\title{
Potential therapy for severe insulin resistance
}

Inhibition of the glucagon receptor in a mouse model of severe insulin resistance normalizes blood levels of glucose, according to a new study. This finding could lead to new treatments for patients with insulin-resistance syndromes.

"Patients with inactivating mutations in the insulin receptor or in components of the downstream signalling pathways are severely diabetic, which in part results from increased hepatic glucose production," explains corresponding author Jesper Gromada. The investigators, therefore, hypothesized that blocking the action of glucagon, which promotes hepatic glucose output, could be effective in these patients.

To test this idea, Gromada and co-workers developed a mouse model of severe insulin resistance.
They then inhibited the glucagon receptor with a monoclonal antibody. Blood levels of glucose normalized within days of the monoclonal antibody being administered. "An unexpected finding was that pancreatic $\beta$-cell mass increased significantly more when the glucagon receptor blocking antibody was administrated to the mice," says Gromada.

In a second paper, Gromada and colleagues demonstrated that, although ANGPTL4 is involved in regulating plasma levels of triglycerides, it is not implicated in the increase in glucagon levels that occurs when the glucagon receptor is inhibited. The authors provide additional evidence in humans that amino acids have an important role in regulating glucagon secretion and $\alpha$-cell mass.
The investigators are now planning a clinical trial to test the use of the glucagon receptor blocking antibody in patients. They are also hoping to determine how blocking the glucagon receptor resulted in increased $\beta$-cell mass in the mice. "Our findings suggest that blocking glucagon action in patients with severe insulin resistance might be efficacious and provide the first treatment option to these patients," concludes Gromada.

Claire Greenhill

ORIGINAL ARTICLES Okamoto, H. et al.

Glucagon receptor inhibition normalizes blood glucose in severe insulin-resistant mice. Proc. Natl Acad. Sci. USA http://dx.doi.org/10.1073/ pnas.1621069114 (2017) | Okamoto, H. et al. Angptl4 does not control hyperglucagonemia or $\alpha$-cell hyperplasia following glucagon receptor inhibition. Proc. Natl Acad. Sci. USA http://dx.doi.org/10.1073/pnas.1620989114 (2017) 